\title{
Clinical, Virological, and Cytokine Profiles of Children Infected with Dengue Virus during the Outbreak in Southern Vietnam in 2017
}

\author{
Mya Myat Ngwe Tun, ${ }^{1}$ Thi Thu Thuy Nguyen, ${ }^{2}$ Tsuyoshi Ando, ${ }^{1}$ Shyam Prakash Dumre, ${ }^{3}$ Aung Min Soe, ${ }^{1}$ Corazon C. Buerano, \\ Minh Tuan Nguyen, ${ }^{5}$ Nguyen Thanh Nhan Le, ${ }^{6}$ Van Quang Pham, ${ }^{7}$ Thanh Hung Nguyen, ${ }^{8}$ Thi Quynh Mai Le, ${ }^{2}$ \\ Kouichi Morita, ${ }^{1}$ and Futoshi Hasebe ${ }^{1,9 *}$ \\ ${ }^{1}$ Department of Virology, Institute of Tropical Medicine, Nagasaki University, Nagasaki, Japan; ${ }^{2}$ Department of Virology, National Institute of \\ Hygiene and Epidemiology, Hanoi, Vietnam; ${ }^{3}$ Department of Immunogenetics, Institute of Tropical Medicine, Nagasaki University, Nagasaki, \\ Japan; ${ }^{4}$ Research and Biotechnology, St Luke's Medical Center, Quezon City, Philippines; ${ }^{5}$ Dengue Department, Children Hospital No. (1), Ho Chi \\ Minh, Vietnam; ${ }^{6}$ Infectious Disease Department, Children Hospital No. (1), Ho Chi Minh, Vietnam; ${ }^{7}$ ICU Department, Children Hospital No. (1), Ho \\ Chi Minh, Vietnam; ${ }^{8}$ Children Hospital No. (1), Ho Chi Minh, Vietnam; ${ }^{9}$ Center of International Collaboration Research, Nagasaki University, \\ Nagasaki, Japan
}

\begin{abstract}
Dengue virus (DENV) infection is a major cause of morbidity and mortality in Vietnam, and the incidence is higher and more consistent in the southern part of the country. This study investigated the circulation of DENV serotypes, viremia levels, immunological status, and cytokine levels, with disease severities among children infected in 2017 in Ho Chi Minh City, Southern Vietnam. Acute and convalescent serum samples were collected from clinically diagnosed dengue children. They were confirmed to have DENV infection by NS1 antigen, IgM and IgG ELISAs, virus isolation, and conventional and real-time RT-PCR. Measurement of 10 cytokine levels was performed in the serum samples. All the children were dengue IgM positive; $28 \%$ and $72 \%$ of them had primary and secondary DENV infections, respectively, whereas $54 \%$ of those with secondary infection were children with dengue with warning signs and with severe dengue. Any or mixed infection of the four serotypes of DENV RNA was detected in 58 children. Twenty DENV strains (DENV-1 = 16 and DENV-4 =4) were isolated. Levels of IFN- - , TNF- $\alpha$, MCP-1, IL-10, and IL-6 were significantly higher in severe dengue cases. We report the predominance of DENV-1 over other serotypes in the 2017 dengue outbreak in Southern Vietnam. Our data showed that cytokine expressions were correlated with dengue pathogenesis and may help in identifying an effective therapeutic strategy.
\end{abstract}

\section{INTRODUCTION}

The global incidence of dengue (DEN) has grown dramatically in recent decades. ${ }^{1}$ The mosquito-borne dengue viruses (DENVs) of the genus Flavivirus are positive-sense singlestranded RNA viruses and can be transmitted to humans by the bite of infected Aedes mosquitoes. ${ }^{2}$ Infection with any of the DENV serotypes can result in asymptomatic manifestation or a wide range of clinical manifestations, from mild-to-severe dengue infection. ${ }^{3}$ The 2009 WHO criteria classify DEN according to levels of severity: DEN without warning signs (DwoWS), DEN with warning signs (DwWS), and severe DEN (SD). ${ }^{4}$

There are several mechanisms to explain the severe forms of DENV infection. They include antibody-dependent enhancement of infection, virulence of DENV strain, cellmediated immune response, and quantity and type of cytokines during infection. ${ }^{5}$ It has been observed in several studies that secondary DENV infection by a DENV serotype different from the first infecting serotype is more likely to produce severe disease. ${ }^{6-11}$ Severe DEN is a leading cause of serious illness and death among children in some Asian and Latin American countries. ${ }^{2}$ A number of studies have shown cytokine profiles related to DEN pathogenesis. ${ }^{12-14}$ Studies to elucidate cytokine storm hypothesis by analyzing sera of severe DEN patients in Vietnam, India, and Cuba have shown elevated levels of IFN- $\gamma$, TNF- $\alpha$, and IL-10 in patients with an increased severity of dengue infection. ${ }^{15-17}$

Dengue has caused a substantial health and economic burden in Vietnam, with the number of reported cases varying

\footnotetext{
* Address correspondence to Futoshi Hasebe, Department of Virology, Center of International Collaboration research, 1-12-4 Sakamoto, Institute of Tropical Medicine, Nagasaki University, Nagasaki, Japan 852-8523. E-mail: rainbow@nagasaki-u.ac.jp
}

significantly year by year. Between 2007 and 2016, the average number of reported cases per year was $90,844 .{ }^{18}$ Dengue outbreaks tend to be larger and more frequent in the southern provinces, with the incidence of infection typically peaking between June and October. ${ }^{18}$ According to a 2017 dengue report from the National Institute of Hygiene and Epidemiology in Hanoi (Vietnam), the reported number of dengue cases in Southern Vietnam was 81,626 with 32 deaths; 33,729 of these cases with seven deaths were from Ho Chi Minh City. From 2007, DENV-1 was the dominant serotype in dengue outbreaks in Southern Vietnam. However, there has been limited information about cytokine profiles in Vietnamese children during acute and convalescent phases of DENV infection. In this study, we did a clinical, virological, and cytokine analyses of acute- and convalescent-phase serum samples of children with different types of disease severity and infection (primary or secondary) during the dengue outbreak in 2017 in Ho Chi Minh City, Southern Vietnam.

\section{MATERIALS AND METHODS}

Samples, patients, and ethical approval. During the 2017 DEN outbreak, paired acute and convalescent serum samples were collected from 76 clinically diagnosed dengue pediatric patients (aged 0-17 years), who were admitted to Children's Hospital No (1), Ho Chi Minh City, Southern Vietnam. Acute and convalescent serum samples were obtained from days 3 to 7 and days 8 to 19 after the onset of fever, respectively. Laboratory tests such as hematocrit test (Hct), tourniquet test, platelet (PLT) counts, and SD bioline NS1 antigen rapid test were carried out by using acute serum samples. ${ }^{19}$ Based on the clinical findings supported by laboratory tests, patients were diagnosed and classified based on DEN severity according to the criteria of the 2009 WHO guideline (WHO, 
2009). Serum samples were also collected from a healthy control group composed of schoolchildren (aged $<16$ years) who were negative to DEN diagnostic tests (DENV NS1 antigen and $\lg \mathrm{M}$ ) and found to have no other observable diseases. The study was approved by the Ethics Committee of National Institute of Hygiene and Epidemiology in Vietnam (08061924-7).

Serological confirmation. Serological confirmation of DENV infection was performed by the in-house DENV IgM capture ELISA, ${ }^{20}$ detection of NS1 antigen, and in-house DENV IgG indirect and capture ELISA. ${ }^{21,22}$ In the DENV IgM capture ELISA, optical density (OD) was read at $492 \mathrm{~nm}$ and a $\mathrm{P} / \mathrm{N}$ (positive control or sample OD/negative control OD) ratio $\geq 2$ was considered as positive. NS1 antigen from serum samples was detected by DEN NS1 antigen rapid test (SD bioline, Suwon, Korea). The DENV IgG indirect and capture ELISA, and DENV IgG ELISA (Vircell, Granada, Spain) were used to determine primary and secondary DENV infections. ${ }^{21,23} \mathrm{~A}$ sample with a titer $\geq 1: 29,000$ was considered to be from a patient with a secondary DENV infection. ${ }^{21} \mathrm{~A}$ sample with a titer $<1: 29,000$ was considered to be from a patient with a primary DENV infection. ${ }^{21}$

DENV isolation and serotyping. All acute serum samples were inoculated onto Aedes albopictus clone C6/36 mosquito cells contained in flat culture tubes. Infected cells were incubated at $28^{\circ} \mathrm{C}$ for 7 days in Eagle's minimum essential medium supplemented with $2 \%$ fetal calf serum and $0.2 \mathrm{mM}$ of nonessential amino acids. ${ }^{24}$ The infected culture fluid (ICF) from each tube was collected, aliquoted, and stored at $-80^{\circ} \mathrm{C}$ until use.

RNA was extracted from the ICF by using the viral RNA Mini Kit (Quiagen, Hilden, Germany) according to the manufacturer's instruction. To screen the ICF which contained DENV, RT-PCR was carried out using one-step RT-PCR following the manufacturer's instruction (Takara, Shiga, Japan). Conventional RT-PCR was carried out by using primer sets (Supplemental Table S1) for the detection of DENV and another primer set for the determination of specific DENV serotypes (Supplemental Table S1). ${ }^{25,26}$

Quantification of DENV genome levels. Viral RNA was directly extracted from $140 \mu \mathrm{L}$ of the patient's acute serum by using the same kit to extract RNA from the ICF. A volume of $5 \mu \mathrm{L}$ of RNA was used for quantitative real-time RT-PCR (qRT$\mathrm{PCR})$. Amplification of the envelope gene was performed using a total of $20 \mu \mathrm{L}$ of reaction mixture $(5 \mu \mathrm{L}$ of TaqMan master mix, $9 \mu \mathrm{L}$ of nuclease water, $0.3 \mu \mathrm{L}$ of $100 \mathrm{pmol}$ of forward and reverse primers, and $0.4 \mu \mathrm{L}$ of probe with DENV serotypespecific primers, Supplementary Table S2) using TaqMan Fast Virus 1-Step Master Mix (Life Technologies, Carlsbad, CA) and following the protocol from a previous report. ${ }^{27,28}$ Ten-fold serial dilutions of standard CDNA $\left(10^{8}-10^{2}\right.$ genome copies) were applied for quantification of viral genome levels. ${ }^{28}$ The detection limit for viral genome was 100 copies. The viral genome levels were expressed as $\log _{10}$ genome copies $/ \mathrm{mL}$.

Quantification of DENV viremia levels. Focus formation assay was performed to determine DENV viremia levels in patient sera. ${ }^{29}$ Serially diluted serum samples were inoculated onto Vero cells which were then incubated at $37^{\circ} \mathrm{C}$ for 2 hours followed by the overlaying of $1.25 \%$ methylcellulose 4,000 in $2 \%$ FCS MEM. After incubation at $37^{\circ} \mathrm{C}$ for 3 days, the cells were fixed with $4 \%$ paraformaldehyde phosphate buffer and permeabilized with 1\% Nonidet P-40 in phosphate-buffered saline without magnesium and calcium (PBS-). This was followed by sequential adding at 1-hour interval of Blockace, pooled human sera containing high-titered anti-flavivirus IgG and HRP-conjugated goat antihuman IgG (American Qualex, San Clemante, CA). To visualize positive results, the substrate 3,3'-diaminobenzidine tetrahydrochloride (DAB; Wako, Tokyo, Japan) was added. The stained foci of cells were counted under the microscope to calculate focus-forming units per $\mathrm{mL}$ (FFU/mL) for the virus titer.

Identification and quantification of cytokines. IFN- $\gamma$, TNF- $\alpha$, IL-1 $\beta$, IL-12P40, IL-2, IL-6, IL-10, IL-4, MCP-1, and IL-8 levels from acute- and convalescent-phase serum samples of children were measured by using Milliplex Map kit (Human Cytokine/Chemokine Magnetic Bead Panel 60K, EMD Millipore, Billerica, MA) according to the manufacturer's instructions. ${ }^{30}$ Analysis was carried out on the Bio-Plex 200 instrument, and data were analyzed by Bio-Plex Manager software (version 6).

Statistical analysis. Data were analyzed by using SPSS for Windows, version 22.0 (IBM Corp., Armonk, NY). Continuous variables were presented as median (IQR, interquartile range) and categorical variables as absolute number $(n)$ and percentage (\%). The comparison of continuous variables was performed by the Mann-Whitney $U$ test between two groups and the Kruskal-Wallis test among three or more groups, whereas the chi-squared test (or Fisher's exact test, as appropriate) was used to compare the categorical variables. Spearman's correlation was used to examine the correlation between two continuous variables. An alpha level of 0.05 was used for all statistical tests. A two-tailed $P$-value less than 0.05 was considered statistically significant.

\section{RESULTS}

Characteristics of the study population. The characteristics of the study population are shown in Table 1. According to the 2009 WHO criteria, 19 (25\%), 36 (47\%), and 21 (28\%) of the 76 pediatric patients were diagnosed to have DwoWS, DwWS, and SD, respectively. Forty-seven (62\%) of them were males and $29(38 \%)$ were females, with an average age of 8.5 years. Significant difference in age was found between DwoWS, DwWS, and SD groups (Table 1, Figure 1A). The levels of white blood cell (WBC), Hct, and PLT were measured by using acute-phase serum samples of children. No significant differences were found in the WBC count and Hct levels among the three clinical severity groups. However, a significantly higher WBC count was found in the SD group than in the DwoWS and DwWS groups (Figure 1B). Platelet count was significantly lower in the SD group than in the DwoWS and DwWS groups (Figure 1C). A significantly lower count of PLT was found in patients with secondary infection than in those with primary DENV infection (Figure 1D). Significant differences were found in clinical symptoms such as purpura, abdominal pain, hemorrhagic manifestation, respiratory difficulties, ascites, and pleural effusion among the three groups (Table 1).

Serological examination, infecting virus serotypes, and viremia levels. Forty-five of the 76 children were DENV NS1 positive. Sixteen, 21, and eight of them were from the DwoWS, DwDW, and SD groups, respectively (Table 2). Measurement of RNA in serum samples by qRT-PCR showed that 58 of the 76 children were infected with specific DENV serotypes as follows: 37 children with DENV-1, two children with DENV-2, one child with DENV-3, 15 children with DENV-4, one child with both DENV-1 and DENV-3, two children with both DENV-1 and DENV-4, and one child with both DENV-2 and 
TABLE 1

Demographic, clinical, and laboratory information of the study population subgrouped according to different degrees of severity of dengue infection

\begin{tabular}{|c|c|c|c|c|}
\hline \multirow[b]{2}{*}{ Variables } & \multicolumn{3}{|c|}{ Severity grades } & \multirow[b]{2}{*}{$P$-value } \\
\hline & Dwows & DwWs & SD & \\
\hline Number of children & 19 & 36 & 21 & \\
\hline Age (years), median (IQR) & $9(7-13.2)$ & $10(7-13)$ & $8(4.5-8.7)$ & 0.040 \\
\hline Gender (Male:female) & $14: 05$ & $22: 14$ & $11: 10$ & \\
\hline WBC $\left(\times 10^{3}\right)$, median $(\mathrm{IQR})$ & $2.6(2.2-3.4)$ & $3.3(2.6-4.3)$ & $4.3(3.3-4.6)$ & 0.060 \\
\hline Hct (\%), median (IQR) & $38.9(36.6-41.3)$ & $40.4(37.5-44)$ & $36.75(33.9-38.3)$ & 0.461 \\
\hline PLT $\left(\times 10^{3}\right)$, median $(\mathrm{IQR})$ & $110(97.7-122)$ & $98.0(68.0-121)$ & $18.5(13.2-41.2)$ & 0.0001 \\
\hline Torniquet test positive & $2(25.0)$ & $6(75.0)$ & $0(0.0)$ & 0.002 \\
\hline Fever & $19(26.4)$ & $34(47.2)$ & $19(26.4)$ & 0.470 \\
\hline Muscle pain & $11(35.5)$ & $17(54.8)$ & $3(9.7)$ & 0.232 \\
\hline Headache & $4(19.0)$ & $15(71.5)$ & $2(9.5)$ & 0.097 \\
\hline Rash & 7 (19.4) & $19(52.8)$ & $10(27.8)$ & 0.468 \\
\hline Purpura & $14(26.9)$ & $30(57.7)$ & $8(15.4)$ & 0.002 \\
\hline Vomiting & 7 (17.9) & $20(51.3)$ & $12(30.8)$ & 0.340 \\
\hline Nausea & $9(19.1)$ & 25 (53.2) & $13(27.7)$ & 0.270 \\
\hline Joint pain & $16(32.0)$ & $27(54.0)$ & $7(14.0)$ & 0.277 \\
\hline Abdominal pain & $0(0.0)$ & 24 (64.9) & $13(35.1)$ & 0.0001 \\
\hline Haemorrhagic manifestation* & $0(0.0)$ & $8(72.7)$ & $3(27.3)$ & 0.083 \\
\hline Respiratory difficulties & $1(7.1)$ & $4(28.6)$ & $9(64.3)$ & 0.004 \\
\hline Ascites & $0(0.0)$ & $1(12.5)$ & 7 (87.5) & 0.001 \\
\hline Pleural effusion & $0(0.0)$ & 2 (18.2) & $9(81.8)$ & 0.0001 \\
\hline
\end{tabular}

WBC = white blood cell; Hct = hematocrit test; PLT = platelets; DwoWS = dengue without warning signs; DwWS = dengue with warning signs; $\mathrm{SD}=$ severe dengue. Numbers in the parentheses indicate percentage, unless stated otherwise. All comparisons for categorical variables between/among the groups are performed by using the chi-square test or Fisher's exact test as appropriate, whereas the comparison for continuous variables is performed by using the Kruskal-Wallis test (for more than two groups) and the Mann-Whitney $U$ test (between two groups). $P<0.05$ is considered shereas theco

DENV-4. All of the $76(100 \%)$ children were DENV IgM positive, whereas 21 (28\%) and 55 (72\%) of them had primary and secondary infections, respectively. Among those with secondary infection, 41 children had DwWS and SD, whereas 12 children had DwoWS. No significant difference was found among the three groups in terms of the type of infection (primary and secondary DENV infections) and DENV RNA detection (Table 2).

Dengue virus strains were isolated from 20 children who, with the exception of one, were NS1 positive (Table 3). Of the 20 DENV isolates, 16 were DENV-1, six of which were from
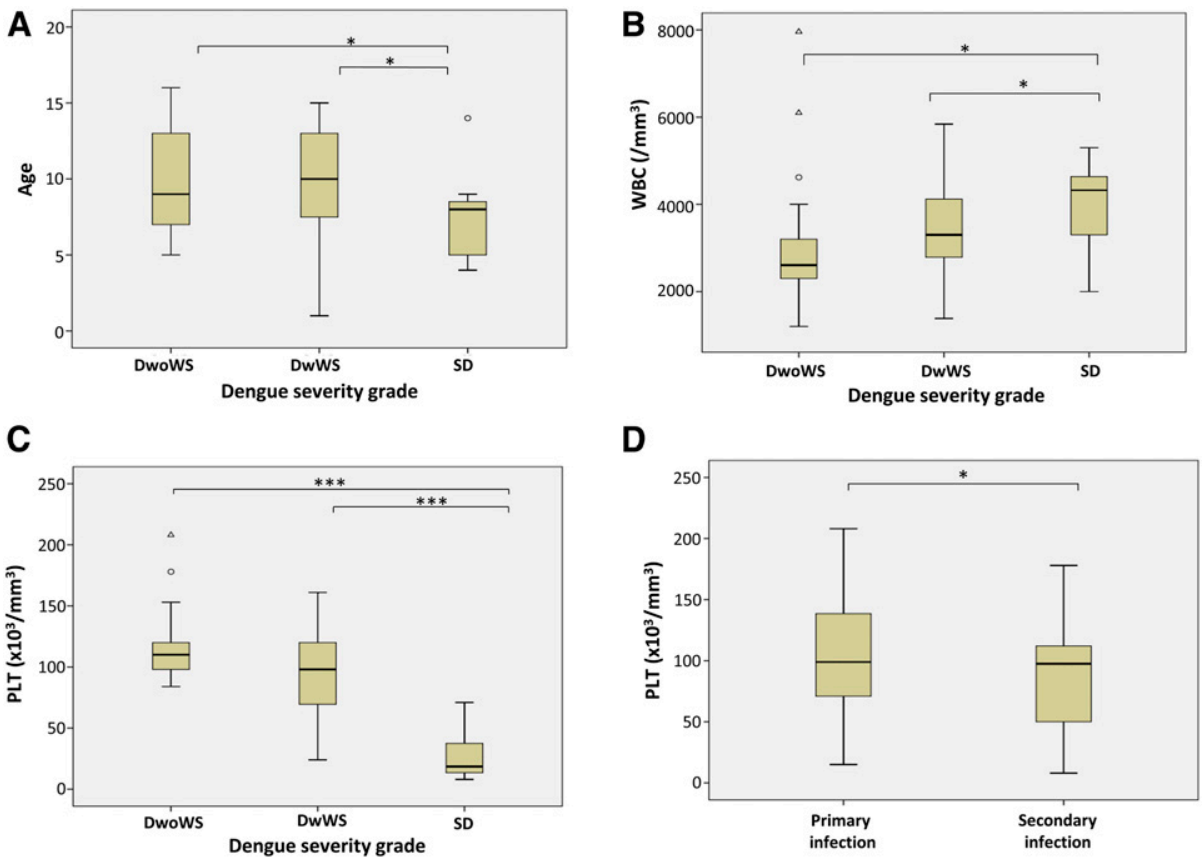

FIGURE 1. Distribution of age of patients and values of hematological parameters according to the different degrees of dengue severity or types of infection. All comparisons among the three groups of children are performed by using the Kruskal-Wallis test. ${ }^{*}$ Comparison between two groups is performed by using the Mann-Whitney $U$ test (A-D). $P$-value less than 0.05 is considered as statistically significant $\left({ }^{\star} P<0.05 ;{ }^{\star \star} P<0.01 ;{ }^{\star \star \star} P<\right.$ 0.001). Box plots show median values (horizontal line in the box), $25-75 \%$ interquartile range (lower-upper limits of the box), additional upper and lower whiskers (represent data outside the IQR including the maximum and minimum values), and outliers (circles or triangles for extreme). DwoWS = dengue without warning signs; DwWS = dengue with warning signs; SD = severe dengue; WBC = white blood cell; PLT = platelets. This figure appears in color at www.ajtmh.org. 
TABLE 2

Diagnostic profiles of children with different dengue severity grades in Vietnam, 2017

\begin{tabular}{|c|c|c|c|c|c|}
\hline \multirow[b]{2}{*}{$\begin{array}{l}\text { Laboratory diagnosis } \\
\text { assays }\end{array}$} & \multirow[b]{2}{*}{ Total } & \multicolumn{3}{|c|}{ Severity grades } & \multirow[b]{2}{*}{$P$-value } \\
\hline & & Dwows & DwWs & SD & \\
\hline DENV NS1 Ag positive & 45 & $16(35.6 \%)$ & $21(46.7 \%)$ & $8(17.8 \%)$ & 0.011 \\
\hline \multicolumn{6}{|l|}{ Virus Isolation } \\
\hline DENV-1 & 16 & $6(37.5 \%)$ & $10(62.5 \%)$ & 0 & 0.648 \\
\hline Primary & 6 & 2 & 4 & 0 & \\
\hline Secondary & 10 & 4 & 6 & 0 & \\
\hline DENV-4 & 4 & $2(50.0 \%)$ & $2(50.0 \%)$ & 0 & - \\
\hline Secondary & 4 & 2 & 2 & 0 & \\
\hline \multicolumn{6}{|l|}{ DENV RNA detection* } \\
\hline DENV-1 & 40 & 9 & 19 & 12 & 0.549 \\
\hline DENV-2 & 3 & 2 & 0 & 1 & \\
\hline DENV-3 & 1 & 0 & 1 & 0 & \\
\hline DENV-4 & 18 & 4 & 8 & 6 & \\
\hline DENV IgM positive & 76 & 19 & 36 & 21 & - \\
\hline \multicolumn{6}{|l|}{ DENV IgG detection } \\
\hline Primary & 21 & $5(23.8 \%)$ & $12(57.1 \%)$ & $4(19.0 \%)$ & 0.540 \\
\hline Secondary & 55 & $14(25.5 \%)$ & $24(43.6 \%)$ & 17 (30.9\%) & - \\
\hline
\end{tabular}

DENV serotypes are detected by real-time RT-PCR. All comparisons for categorical variables between/among the groups are performed by using the chi-square test or Fisher's exact test as is considered significant.

patients with DwoWS and 10 from DwWS. The six patients had primary infection, and 10 had secondary infections, respectively. Four DENV-4 isolates were from two patients with DwoWS, and two from patients with DwWS. These four patients had secondary infection. Two children (sample ID 14 and 20) with DENV-1 isolates were detected by real-time PCR to have RNA from both DENV-1 and DENV-4. One child (sample ID 1) with DENV-4 isolate had DENV RNA levels from both DENV-2 and DENV-4 (Table 3). We measured the DENV genome level $\left(\log _{10}\right.$ copies $\left./ \mathrm{mL}\right)$ and viremia levels $(\mathrm{FFU} / \mathrm{mL})$ using serum samples of children with DENV isolates. Because of volume limitation, virus titration was performed starting with a 100-time dilution to each of the 20 serum samples with DENV isolates. Of the $20 \mathrm{DENV}$ isolates, viremia levels were detected only in 13 children, and seven samples were found to have an undetectable DENV level $(<100)$ (Table 3).

Age distribution. The distribution of children from different age-groups as to the types of infection showed that seven and five children in the 1- to 4-year-old age-group, five and 20 children in the 5- to 8-year-old age-group, five and 13 children in the 9- to 12-year-old age-group, and three and 15 children in the 13- to 16-year-old age-group had primary and secondary infections, respectively, in each age-group. The most number of affected children was in the 5- to 8-year-old age-group with secondary infection (Figure 2). Although the association between age-group and immune response (primary/secondary infection) is not statistically significant $(P=0.057)$, there is a clear trend particularly of increasing secondary infection

TABLE 3

Characteristics of DENV strains isolated from children with different clinical severities and types of DENV infection in Vietnam in 2017

\begin{tabular}{|c|c|c|c|c|c|c|c|c|}
\hline \multirow[b]{2}{*}{ ID } & \multirow[b]{2}{*}{ Clinical severity } & \multirow[b]{2}{*}{ Type of infection } & \multirow[b]{2}{*}{ NS1 } & \multirow[b]{2}{*}{ Virus isolation serotype } & \multicolumn{3}{|c|}{ Real-time PCR (log 10 genome copies $/ \mathrm{mL}$} & \multirow[b]{2}{*}{$\begin{array}{c}\text { Virus titer } \\
\left(\log _{10} \mathrm{FFU} / \mathrm{mL}\right)\end{array}$} \\
\hline & & & & & DENV-1 & DENV-2 & DENV-4 & \\
\hline 1 & DWoWS & $\mathrm{Sec}$ & + & DENV-4 & - & $4.1^{\star}$ & $4.3^{*}$ & 2.9 \\
\hline 2 & DwWS & $\mathrm{Sec}$ & + & DENV-1 & 5.9 & - & - & 3.8 \\
\hline 3 & DwWS & Pri & + & DENV-1 & 5.8 & - & - & 3.8 \\
\hline 4 & DWoWS & $\mathrm{Sec}$ & + & DENV-4 & - & - & 5.1 & - \\
\hline 5 & DwWS & $\mathrm{Sec}$ & + & DENV-1 & 4.0 & - & - & - \\
\hline 6 & DwWS & $\mathrm{Sec}$ & + & DENV-1 & - & - & - & - \\
\hline 7 & DWoWS & $\mathrm{Sec}$ & + & DENV-1 & 6.1 & - & - & 4.7 \\
\hline 8 & DwWS & $\mathrm{Sec}$ & + & DENV-1 & 5.9 & - & _- & 4.5 \\
\hline 9 & DwWS & $\mathrm{Sec}$ & + & DENV-4 & - & - & 5.8 & - \\
\hline 10 & DwWS & $\mathrm{Sec}$ & - & DENV-1 & 5.5 & - & - & - \\
\hline 11 & DwWS & Pri & + & DENV-1 & 5.1 & - & - & 2.9 \\
\hline 12 & DwWS & $\mathrm{Sec}$ & + & DENV-1 & 6.3 & - & - & 4.4 \\
\hline 13 & DWoWS & $\mathrm{Sec}$ & + & DENV-1 & 6.0 & - & - & 2.7 \\
\hline 14 & DWoWS & Pri & + & DENV-1 & $8.0^{\star}$ & - & $3.9^{*}$ & - \\
\hline 15 & DwWS & $\mathrm{Sec}$ & + & DENV-4 & - & - & 7.3 & 4.8 \\
\hline 16 & DwWS & Pri & + & DENV-1 & 7.8 & - & - & 7.0 \\
\hline 17 & DWoWS & Pri & + & DENV-1 & 5.7 & - & - & 3.1 \\
\hline 18 & DwWS & Pri & + & DENV-1 & 7.7 & - & - & 5.9 \\
\hline 19 & DWoWS & $\mathrm{Sec}$ & + & DENV-1 & - & - & 4.7 & - \\
\hline 20 & DWoWS & $\mathrm{Sec}$ & + & DENV-1 & $6.1^{\star}$ & - & $4.3^{*}$ & 3.1 \\
\hline
\end{tabular}




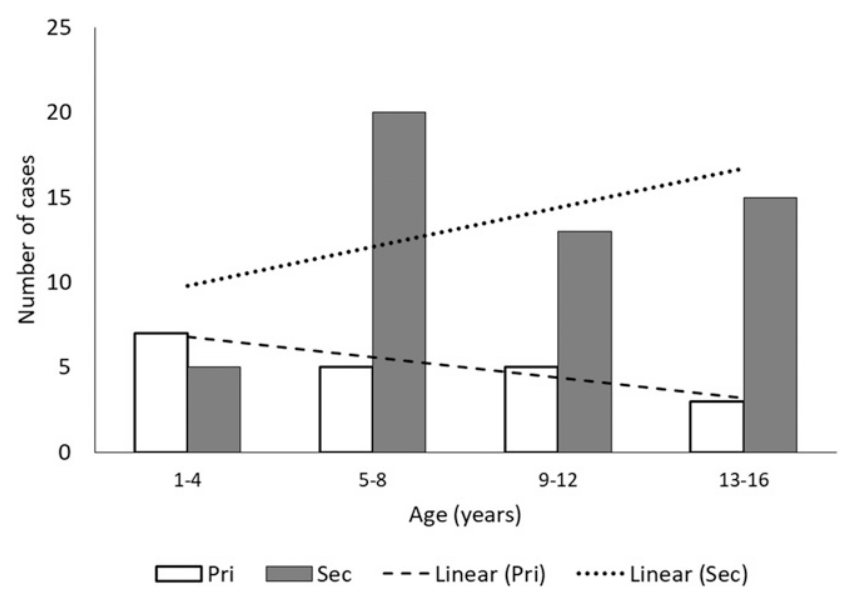

FIGURE 2. Age distribution of children with primary or secondary infection.

with the increasing age and decreasing primary infection with the increasing age (Figure 2).

Pro-inflammatory cytokine profile and clinical parameters. Serum samples from only 58 of the 76 dengue-positive children and from a group of nine healthy children (control) were subjected to cytokine analysis (Figures 3 and 4). Because of inadequate volume, serum samples from 18 of 76 dengue-positive children were not included. Of the 58 children 17, 22, and 19 belonged to the DwoWS, DwWS, and SD groups, respectively. Ten different cytokines were measured: pro-inflammatory (IFN- $\gamma$, TNF- $\alpha$, IL-1 $1 \beta$, IL-12P40, and IL-2), anti-inflammatory (IL-10), pro- and anti-inflammatory cytokines (IL- 6 and IL-4), and two chemokines (MCP-1 and IL-8). The IFN-y levels were significantly higher in both acuteand convalescent-phase serum samples of patients belonging to the three clinical severity groups than in the control group (Figure 3A). The IFN-y levels in the convalescentphase serum samples of the DwWS and SD groups were significantly higher than those of the DwoWS group. The IFN$Y$ levels in the acute-phase serum samples of the DwoWS and DwWS groups were significantly higher than those in the convalescent phase. The TNF- $\alpha$ levels were significantly higher in the acute-phase serum samples of the DwWS group and in both acute- and convalescent-phase serum samples of the SD group than those of the control group (Figure 3B). During the convalescent phase, TNF- $\alpha$ levels were significantly increased in the SD group as compared with the DwoWS and DwWS groups. The levels of IL-1 $\beta$ were significantly increased in the convalescent-phase serum samples of the DwWS group compared with those of the control group (Figure 3C). IL-12P40 levels were significantly higher in both phases of serum samples of patients in the three clinical severity groups than those of the control group (Figure 3D). There were no detectable IL-2 levels in both the three dengue severity groups and the control group.

Anti-inflammatory and pro- and anti-inflammatory cytokines, chemokine profiles, and clinical parameters. The levels of pro- and anti-inflammatory cytokine IL-6 were significantly higher in the acute-phase serum samples of the DwWS group and in both phases of serum samples of the SD group than the control group (Figure 4A). IL-6 was significantly increased in both phases of serum samples of the SD group compared with the DwoWS and DwWS groups. The levels of
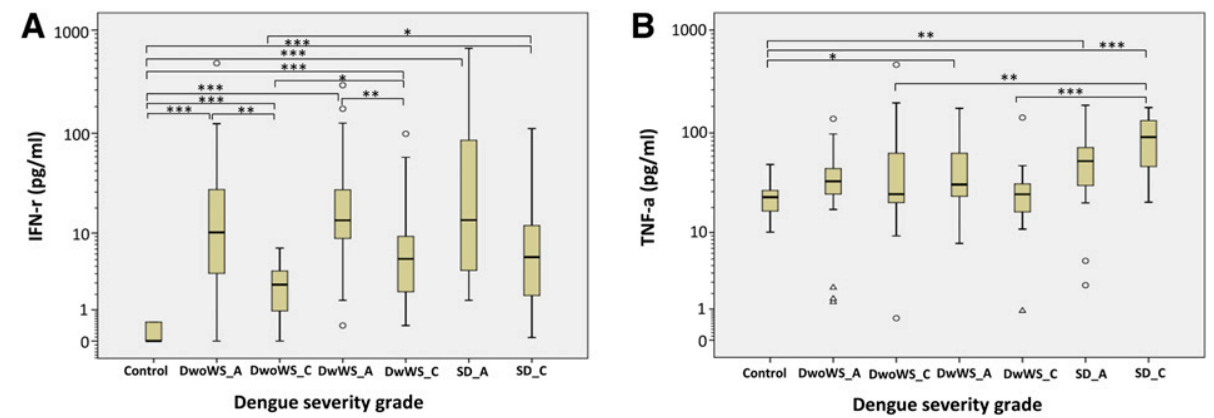

C

D
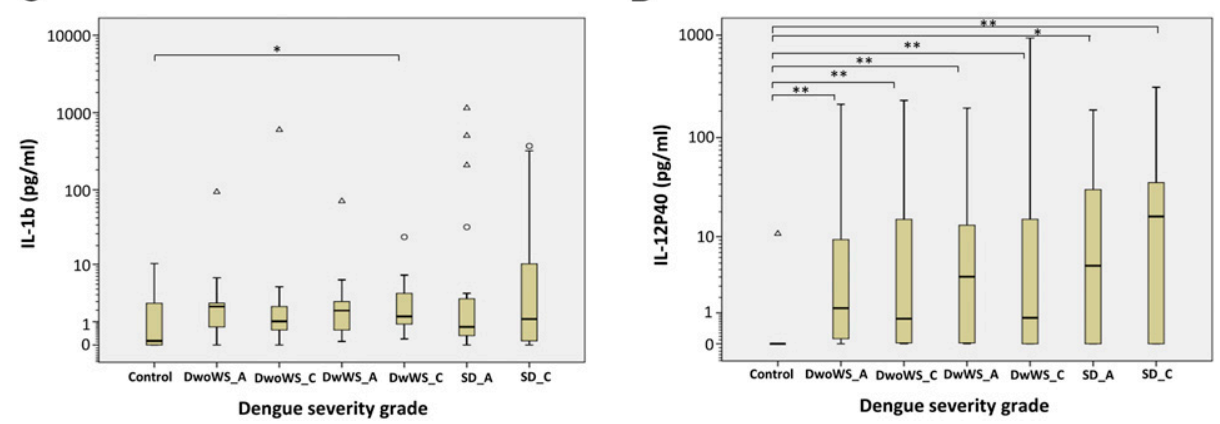

FIGURE 3. Serum levels of pro-inflammatory cytokines in children with different degrees of dengue severity compared with healthy controls. All comparisons among three or more groups are performed by using the Kruskal-Wallis test. *Comparison between two groups is performed by using the Mann-Whitney $U$ test. $P$-value less than 0.05 is considered as statistically significant $\left({ }^{\star} P<0.05 ;{ }^{\star \star} P<0.01 ;{ }^{* \star \star} P<0.001\right)$. Box plots show median values (horizontal line in the box), 25-75\% interquartile range (lower-upper limits of the box), additional upper and lower whiskers (represent data outside the IQR including the maximum and minimum values), and outliers (circles or triangles for extreme). DwoWS-A,C = acute and convalescent phase of dengue without warning signs ( $n=17)$; DwWS-A,C = acute and convalescent phase of dengue with warning signs $(n=22)$; SD-A,C = acute and convalescent phase of severe dengue $(n=19)$. This figure appears in color at www.ajtmh.org. 

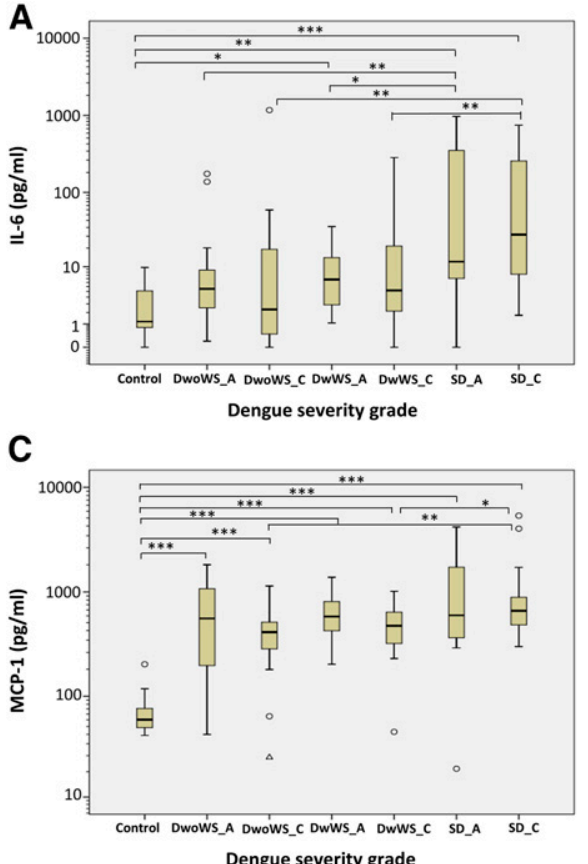

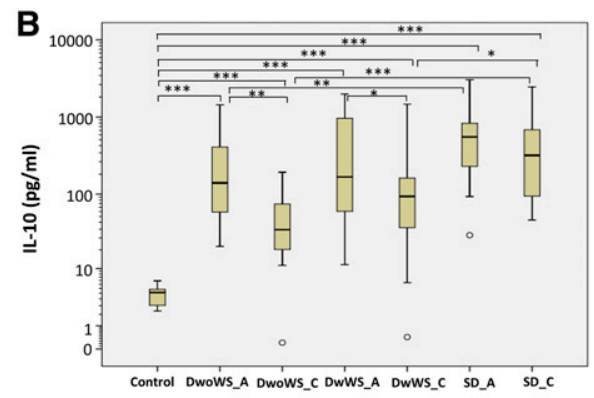

Dengue severity grade

D

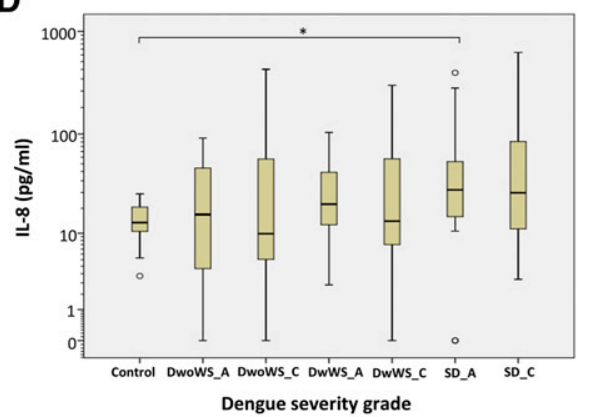

FIGURE 4. Serum levels of anti-inflammatory cytokines in children with different degrees of dengue severity compared with healthy controls. All comparisons among three or more groups are performed by using the Kruskal-Wallis test. ${ }^{*}$ Comparison between two groups is performed by using the Mann-Whitney $U$ test. $P$-value less than 0.05 is considered as statistically significant $\left({ }^{*} P<0.05 ;{ }^{\star \star} P<0.01 ;{ }^{\star \star \star} P<0.001\right)$. Box plots show median values (horizontal line in the box), 25-75\% interquartile range (lower-upper limits of the box), additional upper and lower whiskers (represent data outside the IQR including the maximum and minimum values), and outliers (circles or triangles for extreme). DwoWS-A,C=acute and convalescent phase of dengue without warning signs $(n=17)$; DwWS-A,C = acute and convalescent phase of dengue with warning signs $(n=22)$; SD-A,C = acute and convalescent phase of severe dengue $(n=19)$. This figure appears in color at www.ajtmh.org.

anti-inflammatory cytokine IL-10 were significantly higher in both phases of serum samples of the three clinical severity groups than those of the control group (Figure 4B). Particularly, the IL-10 levels were significantly higher in the SD group than in the DwoWS group during the acute phase of infection. Moreover, IL-10 level in convalescent-phase serum samples of the SD group was significantly higher than that of the DwoWS and DwWS groups. In the DwoWs and DwWS groups, IL-10 levels were significantly higher in the acutephase than in the convalescent-phase serum samples. The levels of chemokine MCP-1 were significantly higher in both phases of the serum samples of the DwoWS, DwWS, and SD groups than those of the control group (Figure 4C). During the convalescent phase, MCP-1 levels in the SD group were significantly higher than those in the DwoWS and DwWS groups. The levels of chemokine IL-8 were significantly higher in acute-phase serum samples of the SD group than those in the control group (Figure 4D). The level of pro- and antiinflammatory cytokine IL-4 productions in the dengue groups of children and the control group was mostly undetectable. The $P$-values on levels of pro- and anti-inflammatory cytokines in children having DwoWS, DwWS, SD, and healthy controls are described in Supplemental Table S3.

Association of pro-inflammatory, anti-inflammatory, pro- and anti-inflammatory cytokines and chemokines with primary and secondary DENV infections. We compared the cytokines and chemokine levels among patients with primary DENV infection, secondary DENV infection, and healthy controls (Table 4). During the acute phase, the levels of IFN- $\gamma$, IL-6, IL-10, and MCP-1 were significantly higher in patients with primary and secondary infections than those in healthy controls. Similarly, the levels of IFN-y, IL-6, IL-10, MCP-1, and IL-12P40 were significantly higher in patients with primary and secondary infections than those in healthy controls during the convalescent phase. Notably, levels of IFN-Y and IL-6 in convalescent sera were significantly higher in patients with secondary infection than those in patients with primary infection.

\section{DISCUSSION}

Our study described the serological, molecular, and immunological status of children infected with DENV during the dengue outbreak in 2017 in Ho Chi Minh City, Southern Vietnam. All patients in this study were DENV IgM positive; $27.6 \%$ and $72.3 \%$ of them had primary and secondary DENV infections, respectively. Similar to the previous studies involving Vietnamese children and adults, the number of patients with secondary infection in this study was higher than that of those with primary infection. ${ }^{31-33}$ In the present study, there were more patients with secondary infection in DwWS and SD groups than those with primary infection. Other studies have also shown that severe DENV infection is chiefly seen in secondary infection. ${ }^{7,34}$ In our study, the number of NS1positive patients was significantly higher in the DwoWS and DwWS groups than that of those in the SD group. A total of 20 DENV were isolated from DwoWS and DwWS patients, 19 of whom were NS1 positive. The less number of NS1 (8 of 21) positive and no DENV isolation among the SD group might be because of collection of blood at a later time after the onset of illness because patients sought primary health care first before going to a referral hospital. 
TABLE 4

Comparison of cytokines and chemokine levels in acute and convalescent sera of children with primary and secondary infections, Vietnam, 2017

\begin{tabular}{|c|c|c|c|c|}
\hline Cytokine or chemokine & Control $(N=9)$ & Primary infection $(N=15)$ & Secondary infection $(N=43)$ & $P$-value \\
\hline \multicolumn{5}{|l|}{ Acute sera } \\
\hline IFN-y & $0.0(0.0-0.5)$ & $8.7(3.5-16-8)$ & $14.3(5.6-79.8)$ & 0.0001 \\
\hline IL-10 & $4.3(2.5-5.2)$ & $155.2(36.3-409.2)$ & $332-7$ (102.5-928.6) & 0.001 \\
\hline IL-12P40 & $0.0(0.0-0.0)$ & $6.8(0.1-20.1)$ & $1.9(0.0-18.9)$ & 0.08 \\
\hline IL-1b & $0.1(0.0-2.4)$ & $1.2(0.7-2.3)$ & $1.5(0.4-3.8)$ & 0.18 \\
\hline IL-6 & $1.1(0.7-6.3)$ & $5.3(2.5-17.2)$ & $7.3(2.8-22.1)$ & 0.024 \\
\hline IL-8 & $12.9(7.9-19.6)$ & $18.2(12.0-49.2)$ & $22.1(7.4-46.2)$ & 0.31 \\
\hline MCP-1 & $59.3(45.8-97.2)$ & $687.2(203.0-1,179.9)$ & 558.0 (381.7-992.4) & 0.0001 \\
\hline TNF- $\alpha$ & $23.2(16.4-27.3)$ & $34.1(22.2-86.7)$ & $34.8(25.3-63.3)$ & 0.07 \\
\hline \multicolumn{5}{|l|}{ Convalescent sera } \\
\hline IFN- $\gamma$ & $0.0(0.0-0.5)$ & $5.9(3.5-12.9)$ & $3.2(1.1-6.8)$ & $0.0001^{*}$ \\
\hline IL-10 & $4.3(2.5-5.2)$ & 94.4 (74.3-144.2) & $75.3(29.0-332.1)$ & 0.0001 \\
\hline IL-12P40 & $0.0(0.0-0.0)$ & $4.2(0.0-42.1)$ & $1.0(0.0-24.0)$ & 0.011 \\
\hline IL-1b & $0.1(0.0-2.4)$ & $1.3(0.5-4.6)$ & $1.1(0.4-2.9)$ & 0.15 \\
\hline IL-6 & $1.1(0.7-6.3)$ & $30.5(3.7-59.4)$ & $5.6(1.1-27.6)$ & $0.013^{\star}$ \\
\hline IL-8 & $12.9(7.9-19.6)$ & $37.5(8.7-86.1)$ & $12.9(6.4-56.9)$ & 0.37 \\
\hline MCP-1 & $59.3(45.8-97.2)$ & $471.1(412.5-890.5)$ & 482.8 (321.0-660.6) & 0.0001 \\
\hline TNF- $\alpha$ & $23.2(16.4-27.3)$ & $43.5(25.7-132.2)$ & $30.1(20.4-78.1)$ & 0.08 \\
\hline
\end{tabular}

Cytokine concentrations are expressed in pg/mL. Cytokines and chemokines levels are expressed as median (IQR) for each groups (control, primary, and secondary infection). All comparisons within groups are performed by using the Kruskal-Wallis test, and comparison between two groups is performed by the Mann-Whitney test. Range of numbers in parentheses beside the median refers to minimum-maximum values.

${ }^{\star}$ Between patients with primary and secondary infections, level of IFN-r $(P=0.02)$ and IL-10 $(P=0.04)$ are significantly different.

As DENV qRT-PCR results showed, the most dominant serotype was DENV-1 (52.6\%), followed by DENV-4 (23.6\%), DENV-2 (4.8\%), and DENV-3 (1.3\%). Based on virus isolation results, DENV-1 (21\%) was dominant, followed by DENV-4 (5.2\%). Interestingly, DENV-1 was the dominant serotype in the 2017 dengue outbreak and had persistently dominated for about one decade in Southern Vietnam. For further studies, a molecular analysis and phenotypic and genotypic characterization will be carried out for DENV-1 and DENV-4 isolates in this study.

IFN- $\mathrm{Y}$ is produced by $\mathrm{T}$ cells and NK cells and activated monocytes and macrophages. ${ }^{35}$ In our study, increased production of IFN- $y$ levels was observed during the acute phase of infection of the SD group, compared with the DwoWS and DwWS groups. High levels of IFN-Y were observed in dengue patients from Asia and Latin America and were associated with severity. ${ }^{36}$ Similar levels of IFN-y were found in the DwoWS and the DwWS groups in the present study, and these results were similar with those of another study in Thai children. ${ }^{37}$ TNF- $\alpha$ is secreted by monocytes, and it enhances vascular permeability and coagulationactivating effects. ${ }^{12}$ TNF- $\alpha$ has been reported to be associated with severity and has higher concentration values for patients with dengue haemorrhagic fever than those with dengue fever in Brazil and Thailand. ${ }^{12,38-40}$ Our finding showed that TNF- $\alpha$ was significantly increased in the DwsWS and SD groups compared with the controls. IL-1 $\beta$ is released by mononuclear monocytes activation with IFN-y. ${ }^{32}$ Studies have shown that significant increase or highest level of IL-1 $\beta$ occurs in SD patients compared with those with mild dengue in Brazil and Vietnam. ${ }^{32,41}$ However, our result showed that the IL-1 $\beta$ level was not significantly different among the DwoWS, DwWS, SD, and control groups.

IL-12 induces the production of IFN-y by T or NK cells. ${ }^{42}$ In the present study, the role of IL-12 in DENV infection was assessed by measuring the circulating levels of the p40subunit of IL-12 (IL-12 p40). A significant increased level of IL$12 p 40$ was observed in the clinical severity groups compared with controls. However, in another study, no significant IL-12- p40 response was observed in dengue patients, compared with controls. ${ }^{43}$ The concentration levels of some cytokines such as IL-1ß, IL-8, and IL-12P40 were not significantly different among DwoWS, DwWS, and SD groups in our study. IL6 , pro- and anti-inflammatory cytokine, is secreted by $T$ cells, macrophages, and NK cells and influences antigen-specific immune responses and inflammatory reactions. ${ }^{12}$ Some studies have described increased levels of IL-6 in SD versus DwoWS and control groups, whereas others failed to show the same results. ${ }^{12,31,41,44}$ In our study, IL-6 levels were significantly higher in SD versus the DwoWS and DwWS groups during the acute phase of infection.

With IL-10, other studies have shown increased level in the DwWS and SD groups compared with the DwoWS group. ${ }^{17,45}$ IL-10 has been shown to be associated with other virus infections such as influenza and to be related to neurological complications. ${ }^{46}$ Our findings confirmed the higher IL-10 level in the SD group versus the other two clinical severity groups. In the case of MCP-1, a significant difference was noted in the three severity groups compared with the controls, and the highest MCP-1 level was found in the SD group. This MCP1 chemokine has been associated with permeability changes in endothelial cells. ${ }^{47}$ Chemokine IL-8, which is produced by monocytes, endothelial cells, and hepatocytes, enhances vascular permeability and activates the innate immune system. Elevated levels of IL-8 have been observed in DSS patients compared with not-severe dengue patients and healthy controls. ${ }^{48,49}$ Our results also showed similar results, as IL-8 levels being significantly higher in the SD group than in the control group.

It has been shown that the DENV nonstructural protein NS1 elicits inflammatory cytokine production and that the endothelial cell monolayer leaks via Toll-like receptor four activation of mouse macrophages and human peripheral blood mononuclear cells. ${ }^{50-52}$ Dengue NS1 has been noted to be directly toxic, damaging endothelial cells and producing lethal vascular permeability in mouse models. ${ }^{52,53}$ Furthermore, antidengue NS1 compounds and antibodies prevent this toxic damage. ${ }^{53}$ Because of the limitation of serum volume in our 
study, we were not able to measure NS1 level and analyze its correlation with the inflammatory cytokines and disease severity. Further studies are needed to clarify cytokine hypothesis to severe dengue pathogenesis.

In conclusion, we report the predominance of DENV-1 over other serotypes in the 2017 dengue outbreak in Southern Vietnam. Our study showed that the levels of IFN- $\gamma$, TNF- $\alpha$, MCP-1, IL-10, and IL-6 were significantly higher in children with severe dengue. The findings support monitoring of changes in the characteristics of the circulating DENV to generate more robust epidemiological data. The cytokine profiles from this study could provide future discovery and validation of biomarkers for the prediction of severe dengue infection.

Received August 16, 2019. Accepted for publication February 9, 2020.

Published online March 16, 2020.

Note: Supplemental tables appear at www.ajtmh.org.

Acknowledgments: We are grateful for the support of the members of the Department of Virology, Institute of Tropical Medicine, Nagasaki University. This work was conducted at the Joint Usage/Research Center on Tropical Disease, Institute of Tropical Medicine, Nagasaki University.

Financial support: This work was supported by grants from the Japan Initiative for Global Research Network on Infectious Diseases (J-GRID, JP19fm0108001) and Research Program on Emerging and Reemerging Infectious Diseases of the Agency for Research and Development (AMED, 19fk0108035h1203).

Authors' addresses: Mya Myat Ngwe Tun, Tsuyoshi Ando, Aung Min Soe, Kouichi Morita, and Futoshi Hasebe, Department of Virology, Institute of Tropical Medicine, Nagasaki University, Nagasaki, Japan, E-mails: myamyat@tm.nagasaki-u.ac.jp, tsuyoshi.ando@miraca.com, dr.aungminnsoe@gmail.com, moritak@nagasaki-u.ac.jp, and rainbow@ nagasaki-u.ac.jp. Thi Thu Thuy Nguyen and Thi Quynh Mai Le, Department of Virology, National Institute of Hygiene and Epidemiology, Hanoi, Vietnam, E-mails: ticun2002@yahoo.com and lom9@ hotmail.com. Shyam Prakash Dumre, Department of Immunogenetics, Institute of Tropical Medicine, Nagasaki University, Nagasaki, Japan, E-mail: sp.dumre@gmail.com. Corazon C. Buerano, Research and Biotechnology, St Luke's Medical Center, Quezon City, Philippines, E-mail: ccbuerano@hotmail.com. Minh Tuan Nguyen, Dengue Department, Children Hospital No (1), Ho Chi Minh, Vietnam, E-mail: tuanmn@nhidong. org.vn. Nguygen Thanh Nhan Le, Infectious Disease Department, Children Hospital No (1), Ho Chi Minh, Vietnam, E-mail: drnhanbvnhidong@ gmail.com. Van Quang Pham, ICU Department, Children Hospital No (1), Ho Chi Minh, Vietnam, E-mail: phamvanquang73@yahoo.com.vn. Than Hung Nguyen, Children Hospital No (1), Ho Chi Minh, Vietnam, E-mail: drthanhhung@gmail.com.

\section{REFERENCES}

1. WHO, 2018. Dengue and Severe Dengue. Available at: https:// www.who.int/news-room/fact-sheets/detail/dengue-andsevere-dengue. Accessed November 4, 2019.

2. Bhatt $S$ et al., 2013. The global distribution and burden of dengue. Nature 496: 504-507.

3. Gubler DJ, 2002. Epidemic dengue/dengue hemorrhagic fever as a public health, social and economic problem in the 21st century. Trends Microbiol 10: 100-103.

4. WHO, 2009. Dengue Guidelines for Diagnosis, Treatment, Prevention, and Control. Geneva, Switzerland: World Health Organization.

5. Restrepo BN, Isaza DM, Salazar CL, Ramirez R, Ospina M, Alvarez LG, 2008. Serum levels of interleukin-6, tumor necrosis factoralpha and interferon-gamma in infants with and without dengue. Rev Soc Bras Med Trop 41: 6-10.

6. Vaughn DW et al., 2000. Dengue viremia titer, antibody response pattern, and virus serotype correlate with disease severity. $J$ Infect Dis 181: 2-9.
7. Burke DS, Nisalak A, Johnson DE, Scott RM, 1988. A prospective study of dengue infections in Bangkok. Am J Trop Med Hyg 38: 172-180.

8. Halstead SB, Nimmannitya S, Yamarat C, Russell PK, 1967. Hemorrhagic fever in Thailand; recent knowledge regarding etiology. Jpn J Med Sci Biol 20 (Suppl): 96-103.

9. Halstead SB, Nimmannitya S, Cohen SN, 1970. Observations related to pathogenesis of dengue hemorrhagic fever. IV. Relation of disease severity to antibody response and virus recovered. Yale J Biol Med 42: 311-328.

10. Russell PK, Yuill TM, Nisalak A, Udomsakdi S, Gould DJ, Winter PE, 1968. An insular outbreak of dengue hemorrhagic fever. II. Virologic and serologic studies. Am J Trop Med Hyg 17: 600-608.

11. Sangkawibha N, Rojanasuphot $S$, Ahandrik $S$, Viriyapongse $S$, Jatanasen S, Salitul V, Phanthumachinda B, Halstead SB, 1984. Risk factors in dengue shock syndrome: a prospective epidemiologic study in Rayong, Thailand. I. The 1980 outbreak. Am J Epidemiol 120: 653-669.

12. Hober $D$ et al., 1993. Serum levels of tumor necrosis factor-alpha (TNF-alpha), interleukin-6 (IL-6), and interleukin-1 beta (IL-1 beta) in dengue-infected patients. Am J Trop Med Hyg 48: 324-331.

13. Fink J, Gu F, Vasudevan SG, 2006. Role of T cells, cytokines and antibody in dengue fever and dengue haemorrhagic fever. Rev Med Virol 16: 263-275.

14. Green $S$ et al., 1999. Early immune activation in acute dengue illness is related to development of plasma leakage and disease severity. J Infect Dis 179: 755-762.

15. Nguyen TH et al., 2004. Dengue hemorrhagic fever in infants: a study of clinical and cytokine profiles. J Infect Dis 189: 221-232.

16. Chakravarti A, Kumaria R, 2006. Circulating levels of tumour necrosis factor-alpha \& interferon-gamma in patients with dengue \& dengue haemorrhagic fever during an outbreak. Indian $J$ Med Res 123: 25-30.

17. Perez $A B$ et al., 2004. IL-10 levels in dengue patients: some findings from the exceptional epidemiological conditions in Cuba. J Med Virol 73: 230-234.

18. Hung TM, Clapham HE, Bettis AA, Cuong HQ, Thwaites GE, Wills BA, Boni MF, Turner HC, 2018. The estimates of the health and economic burden of dengue in Vietnam. Trends Parasitol 34: 904-918.

19. Zhang H, Li W, Wang J, Peng H, Che X, Chen X, Zhou Y, 2014. NS1-based tests with diagnostic utility for confirming dengue infection: a meta-analysis. Int J Infect Dis 26: 57-66.

20. Bundo K, Igarashi A, 1985. Antibody-capture ELISA for detection of immunoglobulin $M$ antibodies in sera from Japanese encephalitis and dengue hemorrhagic fever patients. I Virol Methods 11: 15-22.

21. Inoue $S$ et al., 2010. Evaluation of a dengue IgG indirect enzymelinked immunosorbent assay and a Japanese encephalitis IgG indirect enzyme-linked immunosorbent assay for diagnosis of secondary dengue virus infection. Vector Borne Zoonotic Dis 10: $143-150$.

22. Ngwe Tun MM et al., 2013. Serological characterization of dengue virus infections observed among dengue hemorrhagic fever/ dengue shock syndrome cases in upper Myanmar. $J$ Med Virol 85: 1258-1266.

23. Bocanegra $\mathrm{C}$ et al., 2016. Zika virus infection in pregnant women in Barcelona, Spain. Clin Microbiol Infect 22: 648-650.

24. Ngwe Tun MM et al., 2014. Detection of east/central/south African genotype of chikungunya virus in Myanmar. Emerg Infect Dis 20: 1378-1381.

25. Lanciotti RS, Calisher CH, Gubler DJ, Chang GJ, Vorndam AV, 1992. Rapid detection and typing of dengue viruses from clinical samples by using reverse transcriptase-polymerase chain reaction. J Clin Microbiol 30: 545-551.

26. Morita K, Tanaka M, Igarashi A, 1991. Rapid identification of dengue virus serotypes by using polymerase chain reaction. J Clin Microbiol 29: 2107-2110.

27. Kyaw AK et al., 2017. Clinical, virological and epidemiological characterization of dengue outbreak in Myanmar, 2015. Epidemiol Infect 145: 1886-1897.

28. Ito M, Takasaki T, Yamada K, Nerome R, Tajima S, Kurane I, 2004. Development and evaluation of fluorogenic TaqMan reverse 
transcriptase PCR assays for detection of dengue virus types 1 to 4. J Clin Microbiol 42: 5935-5937.

29. Kinoshita $\mathrm{H}$ et al., 2009. Isolation and characterization of two phenotypically distinct dengue type- 2 virus isolates from the same dengue hemorrhagic fever patient. Jpn J Infect Dis 62: 343-350.

30. Won JH, Goldberger O, Shen-Orr SS, Davis MM, Olshen RA, 2012. Significance analysis of XMap cytokine bead arrays. Proc Natl Acad Sci U S A 109: 2848-2853.

31. Mangione JN et al., 2014. The association of cytokines with severe dengue in children. Trop Med Health 42: 137-144.

32. Chau TN et al., 2008. Dengue in Vietnamese infants-results of infection-enhancement assays correlate with age-related disease epidemiology, and cellular immune responses correlate with disease severity. J Infect Dis 198: 516-524.

33. Thai KT et al., 2010. Clinical, epidemiological and virological features of dengue virus infections in Vietnamese patients presenting to primary care facilities with acute undifferentiated fever. J Infect 60: 229-237.

34. Halstead SB, 1988. Pathogenesis of dengue: challenges to molecular biology. Science 239: 476-481.

35. Schroder K, Hertzog PJ, Ravasi T, Hume DA, 2004. Interferongamma: an overview of signals, mechanisms and functions. J Leukoc Biol 75: 163-189.

36. Azeredo EL, Zagne SM, Alvarenga AR, Nogueira RM, Kubelka CF, de Oliveira-Pinto LM, 2006. Activated peripheral lymphocytes with increased expression of cell adhesion molecules and cytotoxic markers are associated with dengue fever disease. Mem Inst Oswaldo Cruz 101: 437-449.

37. Kurane I, Innis BL, Nimmannitya S, Nisalak A, Meager A, Janus J, Ennis FA, 1991. Activation of T lymphocytes in dengue virus infections. High levels of soluble interleukin 2 receptor, soluble CD4, soluble CD8, interleukin 2, and interferon-gamma in sera of children with dengue. J Clin Invest 88: 1473-1480.

38. Braga EL, Moura P, Pinto LM, Ignacio SR, Oliveira MJ, Cordeiro MT, Kubelka CF, 2001. Detection of circulant tumor necrosis factor-alpha, soluble tumor necrosis factor p75 and interferongamma in Brazilian patients with dengue fever and dengue hemorrhagic fever. Mem Inst Oswaldo Cruz 96: 229-232.

39. Kittigul L, Temprom W, Sujirarat D, Kittigul C, 2000. Determination of tumor necrosis factor-alpha levels in dengue virus infected patients by sensitive biotin-streptavidin enzyme-linked immunosorbent assay. J Virol Methods 90: 51-57.

40. Gagnon SJ, Mori M, Kurane I, Green S, Vaughn DW, Kalayanarooj S, Suntayakorn S, Ennis FA, Rothman AL, 2002. Cytokine gene expression and protein production in peripheral blood mononuclear cells of children with acute dengue virus infections. J Med Virol 67: 41-46.

41. Bozza FA, Cruz OG, Zagne SM, Azeredo EL, Nogueira RM, Assis EF, Bozza PT, Kubelka CF, 2008. Multiplex cytokine profile from dengue patients: MIP-1beta and IFN-gamma as predictive factors for severity. BMC Infect Dis 8: 86 .

42. Kohno K, Kataoka J, Ohtsuki T, Suemoto Y, Okamoto I, Usui M, Ikeda M, Kurimoto M, 1997. IFN-gamma-inducing factor (IGIF) is a costimulatory factor on the activation of Th1 but not Th2 cells and exerts its effect independently of IL-12. J Immunol 158: 1541-1550.

43. Juffrie MM, vd Meer GM, Veerman AJP, Thijs LG, Hack CE, 2002. Inflammatory mediators in dengue virus infection: circulating interleukin-12 and interferon- $y$. Dengue Bull 26: 144-154.

44. Juffrie M, Meer GM, Hack CE, Haasnoot K, Sutaryo, Veerman AJ, Thijs LG, 2001. Inflammatory mediators in dengue virus infection in children: interleukin- 6 and its relation to C-reactive protein and secretory phospholipase A2. Am J Trop Med Hyg 65: 70-75.

45. Green S, Vaughn DW, Kalayanarooj S, Nimmannitya S, Suntayakorn S, Nisalak A, Rothman AL, Ennis FA, 1999. Elevated plasma interleukin-10 levels in acute dengue correlate with disease severity. J Med Virol 59: 329-334.

46. Hasegawa S, Matsushige $T$, Inoue $H$, Shirabe K, Fukano R, Ichiyama T, 2011. Serum and cerebrospinal fluid cytokine profile of patients with 2009 pandemic H1N1 influenza virusassociated encephalopathy. Cytokine 54: 167-172.

47. Sierra B, Perez AB, Vogt K, Garcia G, Schmolke K, Aguirre E, Alvarez M, Volk HD, Guzman MG, 2010. MCP-1 and MIP1alpha expression in a model resembling early immune response to dengue. Cytokine 52: 175-183.

48. Avirutnan P, Malasit P, Seliger B, Bhakdi S, Husmann M, 1998. Dengue virus infection of human endothelial cells leads to chemokine production, complement activation, and apoptosis. J Immunol 161: 6338-6346.

49. Raghupathy $R$ et al., 1998. Elevated levels of IL-8 in dengue hemorrhagic fever. J Med Virol 56: 280-285.

50. Modhiran N, Watterson D, Muller DA, Panetta AK, Sester DP, Liu L, Hume DA, Stacey KJ, Young PR, 2015. Dengue virus NS1 protein activates cells via toll-like receptor 4 and disrupts endothelial cell monolayer integrity. Sci Transl Med 7: 304ra142.

51. Glasner DR, Puerta-Guardo H, Beatty PR, Harris E, 2018. The good, the bad, and the shocking: the multiple roles of dengue virus nonstructural protein 1 in protection and pathogenesis. Annu Rev Virol 5: 227-253.

52. Puerta-Guardo $H$, Glasner DR, Espinosa DA, Biering SB, Patana M, Ratnasiri K, Wang C, Beatty PR, Harris E, 2019. Flavivirus NS1 triggers tissue-specific vascular endothelial dysfunction reflecting disease tropism. Cell Rep 26: 1598.e8-1613.e8.

53. Beatty PR, Puerta-Guardo H, Killingbeck SS, Glasner DR, Hopkins K, Harris E, 2015. Dengue virus NS1 triggers endothelial permeability and vascular leak that is prevented by NS1 vaccination. Sci Transl Med 7: 304ra141. 